\title{
Hexahedral Localization (HL): A Three-Dimensional Hexahedron Localization Based on Mobile Beacons
}

\author{
Linlan Liu, ${ }^{1}$ Haili Zhang, ${ }^{2}$ Xiaotian Geng, ${ }^{3}$ and Xin Shu ${ }^{4}$ \\ ${ }^{1}$ School of Information Engineering, Internet of Things Technology Institute, Nanchang Hangkong University, \\ Nanchang 330063, China \\ ${ }^{2}$ Tianjin Baodi No. 1 Middle School, Tianjin 301800, China \\ ${ }^{3}$ School of Software, Internet of Things Technology Institute, Nanchang Hangkong University, Nanchang 330063, China \\ ${ }^{4}$ School of Electrical Engineering and Computer Science, Washington State University, Pullman, WA 99163, USA
}

Correspondence should be addressed to Linlan Liu; liulinlan@nchu.edu.cn

Received 25 August 2013; Accepted 11 September 2013

Academic Editors: C.-C. Chang and F. Yu

Copyright (C) 2013 Linlan Liu et al. This is an open access article distributed under the Creative Commons Attribution License, which permits unrestricted use, distribution, and reproduction in any medium, provided the original work is properly cited.

\begin{abstract}
In wireless sensor networks, localization is one of the fundamental technologies and is essential to its applications. In this paper, we propose a three-dimensional range-free localization scheme named hexahedral localization. In the scheme, the space is divided into a lot of hexahedrons. Then, all the unknown nodes are located by utilizing the perpendicular properties of the trajectory. The contribution of our scheme can be summarized into two points. First, it fills the gap of shortage of three-dimensional localization based on mobile beacons. Second, it brings in the outstanding localization accuracy. The simulation result reveals that this localization scheme has the relative high accuracy. At the end of the paper, the performance and error of our scheme are analyzed in aim of improving in the future work.
\end{abstract}

\section{Introduction}

Wireless sensor network (WSN) is considered as one of the most influencing technologies in the 21st century and one of the inventions which would change the future world [1]. As the technologies of sensor, microsystem, wireless communication, and the computer developed, the wireless sensor networks are applied more and more widely. In WSN, the location of nodes is significant to the detection. Location information also supports many fundamental network services, including network routing, topology control, coverage, boundary detection, and clustering [2]. So, it is obvious that localization is essential to the applications of wireless sensor network. The localization mechanisms in WSN are usually classified into two categories: range-based mechanisms and range-free mechanisms [1]. Typical range-free localization algorithms include Centroid [3-5], APIT [6-11], and DVHOP [12-14]. They leverage the limited hardware to acquire the location of the nodes with the advantage of low cost and little environmental impact. On the other hand, the rangebased mechanisms, such as TOA [15, 16], TDOA [17-20], and
RSSI [21-25], utilize signal or acoustic wave to get the distance or orientation between nodes in order to calculate the nodes' coordinate.

To sum up, most of the localization mechanisms employ beacon (anchor) nodes and utilize the relationship between the beacons and the unknown node to gain the location of the nodes. However, the beacons should be embedded with the GPS which leads to high hardware cost. In light of this, the researchers propose the localization schemes based on mobile beacons in aim of reducing the cost of hardware. The localization based on mobile beacons utilizes just a few beacons broadcasting the message while moving among the unknown nodes instead of deploying many static beacons. These methods cut down the overhead by a wide margin.

The rest of the paper is organized as follows. Section 2 introduces the related works about the mobile beacon based localization in the past decade. Section 3 gives out the design of the HL. Section 4 is the simulation and result, followed by Section 5 which analyzes the relationship among the parameters. At last, Section 6 represents the error analysis, and we conclude in Section 7. 


\section{Related Works}

During the past decade, the localization schemes based on mobile beacons have been developed in a variety of directions.

The pioneer work can be traced back to 2004. North Carolina State University [26] firstly depicts the initial model of the mobile beacon-based localization. They acquire the node's location via the PDF (probability distribution function) of the estimated position according to the RSSI (received signal strength indicator). Then, they make some remarks regarding two properties that the trajectory should have. At last, the experimental results reveal an unexpectedly good accuracy, almost an order of magnitude better than other static approaches. In 2005, the researcher of National Cheng Kung University, Ssu et al. [27], selects more than 3 beacon points to determine the position of unknown nodes. In the paper, the scheme adopts the RWP (random waypoint), and the paper analyzes the accuracy under different radio ranges of beacon moving speed, and so forth. The result reveals that, as a range-free approach, its accuracy is competitive to other range-based approaches. In 2006, the authors of [27] propose a new localization algorithm based on aerial beacons [28]. It utilizes an aerial beacon moving upon the sensor nodes to locate them via the geometry principles. It is a threedimensional localization algorithm, although it is used in the two-dimensional environment. The simulation shows that its performance is better than other range-free localization schemes. In 2007, Purdue university [29] compared three trajectories of the beacons' movement named Scan, Double Scan, and Hilbert. The result of simulation describes that Scan has the lowest localization error among the three trajectories, followed closely by Hilbert. However, Hilbert is the most robust to the obstacles. In 2008, the Chinese Academy of Science [30] improves the algorithm in [27] by searching for the "maximum RSSI" point as the midpoint of the chord. It gets more reliable reference points to make the accuracy more precise by the ratio of $50 \%$. In 2009 , the Chinese academy of Science [31] further improves the previous scheme through searching for 4 reference points to ensure the position of the unknown nodes. Compared to the previous work, the proposed approach enhances accuracy to a certain degree. In the same year, the Gwangju Institute of Science and Technology [32] improves the algorithm of [27] with the geometric constraints. It points out that the selection of the reference point in [27] is inaccurate and selects 3 noncollinear reference points to locate the nodes with the geometric constraints. As a result, the accuracy is improved. The Chinese National University of Defense and Technology [33] proposed two algorithms on the path planning. These two algorithms are based on graph theory and are called breadth-first and backtracking greedy. The goal of path planning is locating the nodes within less time and cost. In the view of coverage and cost, these two algorithms are effective. In addition, they obtain higher precision and are robust in the environment of the nodes randomly deployed. In 2010, the Ocean University of China [34] proposes a novel localization algorithm based on the mobile beacon. It plans a regular path consisting of equilateral triangles and utilizes the geometric property to locate the unknown nodes. As a latecomer in the part representation arena, this scheme attracts people's eyes. Its design is motivated by the phenomenon between RSSI and straight trajectory of the mobile beacon. The experiment of the scheme with 100 TELOSB motes proves that this scheme is superior to all the existing approaches in terms of high precision.

In 2011, Ou [35] proposes a range-free localization scheme using mobile anchor nodes equipped with four directional antennas. In the proposed approach, each mobile anchor node determines its position via GPS, and then broadcasts its coordinates as it moves through the WSN. The sensor nodes detect these beacon messages and utilize a simple processing scheme to determine their own coordinates based on those of the anchors. It removes the requirement for specific ranging hardware on the sensor nodes and avoids the need for communications between the sensor nodes. In 2012, the INRIA [36] proposed a novel DeteRministic dynamic bEAcon Mobility Scheduling (DREAMS) algorithm, without requiring any prior knowledge of the sensory field. In this algorithm, beacon trajectory is defined as the track of depth-first traversal (DFT) of the network graph, which thus is deterministic. The mobile beacon performed DFT dynamically, under the instruction of nearby sensors on the fly. It moved from sensor to sensor in an intelligent heuristic manner according to received signal strength (RSS) based distance measurements. It was proved that DREAMS guarantees full localization (every sensor is localized) when the measurements are noise free. In the same year, Chang et al. [37] proposed the first study that applies the mobile anchor to improve the location inaccuracy under the condition that all sensors are with different sizes of estimative regions. In 2013, a range-free localization mechanism with ring overlapping by utilizing mobile anchors was proposed by Chen et al. [38]. Since the mobile anchor and the reference node know their own locations, the B-rings, in which the blind node is located, can be precisely derived. Therefore, by overlapping the B-rings, the proposed mechanism can obtain good location estimation for the blind node. Besides, two movement schemes, BTS and ESS, for mobile anchor are also proposed. The proposed scheme has better accuracy than other existing related schemes including ROCRSSI scheme, Centroid scheme, and PBCC scheme.

However, we see that almost all of the algorithms above rarely refer to the three-dimensional localization, since the localization in three-dimensional environments is more complex. According to this blankness, this paper proposes a novel three-dimensional localization scheme based on mobile beacon called HL (hexahedral localization). It is able to locate without any additional hardware and reach the relative high accuracy.

\section{The Design of $\mathbf{H L}$}

In this section, the train of thought about the HL is described. The design of HL is inspired by literature [34]. Firstly, the experiment on RSSI versus Distance is made. Then, we present our new scheme. At last, we optimize the scheme. 
3.1. The Experiment of RSSI versus Distance. As the most popular parameter used in the localization process, RSSI has the advantage of low cost and convenient operation. Theoretically, RSSI obeys the following formula [39]:

$$
\operatorname{PL}(d)=-32.44-20 \log f_{c}-20 \log d .
$$

$\mathrm{PL}(d)$ is the RSSI according to the distance of $d$, and $f_{c}$ is the carrier frequency. From the formula above, we can get that the RSSI decreases as $d$ increases.

Similar literature [34], we observe the interesting regularity. As shown in Figure 1, we deploy 11 TELOSB motes on the campus to observe the RSSI that the node on longitudinal axis receives from which is on the transverse axis. We are surprised to find that the data could plot into a curve as shown in Figure 1. The only difference between our research and literature [34] is the length of the transverse axis. In fact, it is unnecessary to study the width which is too large for the inaccurate RSSI.

As Figure 1 shows, we assume the transverse axis as the trajectory of a mobile beacon. When the mobile beacon tracks along a straight line, the nearest point of the unknown node is the foot point of the trajectory. At the same time, the RSSI is the largest.

Different from literature [34], the purpose of our experiment is to get the suitable distance that the RSSI is available. From Figure 1, we can obtain the trusty distance is $30 \mathrm{~m}$ when the radius is about $50 \mathrm{~m}$. And we continue to do the similar but more accurate experiment under the radius from $40-100 \mathrm{~m}$ by the step of the $10 \mathrm{~m}$. The result is shown in Table 1.

According to Table 1 , the trusty radius analogously equal to $60 \%$ of the radius.

3.2. The Model of the HL. The observation above motivates the design of HL. We project HL as shown in Figure 2.

The mobile beacon moves along the given trajectory and broadcasts its own position periodically. Via RSSI, the unknown node selects the nearest reference points on the trajectory. The coordinate of the reference point is

$$
\left(x_{a}, y_{a}, z_{a}\right)\left(x_{b}, y_{b}, z_{b}\right)\left(x_{c}, y_{c}, z_{c}\right) .
$$

And the unknown node is

$$
(x, y, z) \text {. }
$$

The direction vector of (1), (7), (6) is given as

$$
\left(i_{1}, j_{1}, k_{1}\right)\left(i_{2}, j_{2}, k_{2}\right)\left(i_{3}, j_{3}, k_{3}\right)
$$

Then, we have the following equations:

$$
\begin{aligned}
& i_{1}\left(x-x_{a}\right)+j_{1}\left(y-y_{a}\right)+k_{1}\left(z-z_{a}\right)=0 \\
& i_{2}\left(x-x_{b}\right)+j_{2}\left(y-y_{b}\right)+k_{2}\left(z-z_{b}\right)=0 \\
& i_{3}\left(x-x_{c}\right)+j_{3}\left(y-y_{c}\right)+k_{3}\left(z-z_{c}\right)=0
\end{aligned}
$$

$$
\begin{gathered}
\Longrightarrow M\left(\begin{array}{l}
x \\
y \\
z
\end{array}\right)=N, \\
M=\left(\begin{array}{lll}
i_{1} & j_{1} & k_{1} \\
i_{2} & j_{2} & k_{2} \\
i_{3} & j_{3} & k_{3}
\end{array}\right), \quad N=\left(\begin{array}{l}
i_{1} x_{a}+j_{1} y_{a}+k_{1} z_{a} \\
i_{2} x_{b}+j_{2} y_{b}+k_{2} z_{b} \\
i_{3} x_{c}+j_{3} y_{c}+k_{3} z_{c}
\end{array}\right) .
\end{gathered}
$$

According to the least square method,

$$
\left(\begin{array}{l}
x \\
y \\
z
\end{array}\right)=\left(M^{T} M\right)^{-1} M^{T} N
$$

There must be a lot of errors in the process of calculation via the least square method. We will analyze that in Section 6.

3.3. The Optimization of the Trajectory. We have given out the trajectory of the HL. But how to make the model reasonable is an important issue. From literature [34], it can be known that the equilateral triangle is the best trajectory on the two-dimensional flat. And interestingly, we find that the equilateral triangles can be connected and divided into rectangles like Figure 3. The trajectory we proposed is more controllable at the same time.

As the trajectory described in Section 3.2, according to the radius $R$, the ratio of the hexahedron's edges should be optimized.

In Figure 4, take the red hexahedron, for example, AC, $\mathrm{BD}, \mathrm{HE}, \mathrm{GF}, \mathrm{AE}, \mathrm{BF}, \mathrm{DG}$, and $\mathrm{CH}$ are all the trajectories of the mobile beacon. To ensure the coverage of mobile beacons, the extension that the signal propagates should be equal. In another word, the point in the hexahedron which is furthest from the trajectory should be covered in the extension. According to the trusty radius, the largest distance should be equal to the trusty radius. As the trajectories are deployed symmetrically as shown in Figure 4, we find that the points I, J, K, L are the furthest points to the bevel trajectory like $\mathrm{AC}, \mathrm{BD}, \mathrm{HE}$, and GF. And the point on PQ is the furthest point to the vertical trajectory like $\mathrm{AE}, \mathrm{BF}, \mathrm{DG}$, and $\mathrm{CH}$. We can calculate the distance between the furthest point and the trajectory

$$
\mathrm{IM}=\sqrt{\frac{\mathrm{AE}^{2}}{4}+\frac{\mathrm{AB}^{2} A D^{2}}{\mathrm{AB}^{2}+A D^{2}}}
$$

And the distance from PQ to the trajectory AE, BF, DG, and $\mathrm{CH}$ is

$$
\mathrm{BQ}=\frac{\sqrt{\mathrm{AB}^{2}+\mathrm{AD}^{2}}}{2} .
$$

The distance IM and BQ should be equal to the trusty radius. Then,

$$
R_{\text {trusty }}=\sqrt{\frac{\mathrm{AE}^{2}}{4}+\frac{\mathrm{AB}^{2} \mathrm{AD}^{2}}{\mathrm{AB}^{2}+\mathrm{AD}^{2}}}=\frac{\sqrt{\mathrm{AB}^{2}+\mathrm{AD}^{2}}}{2} .
$$




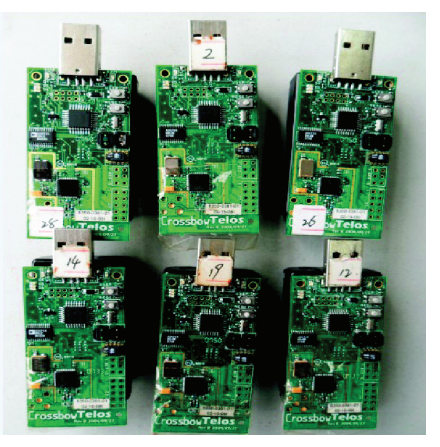

(a)

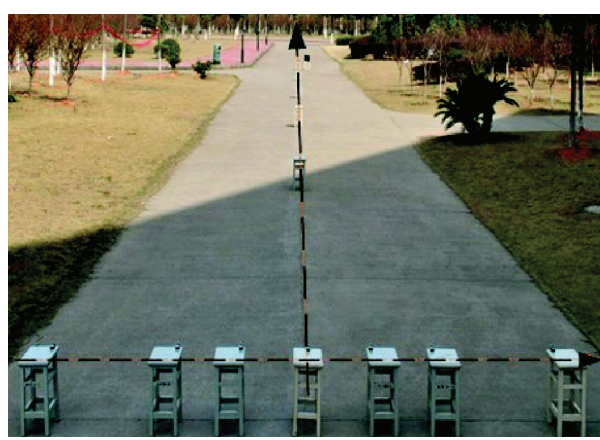

(b)

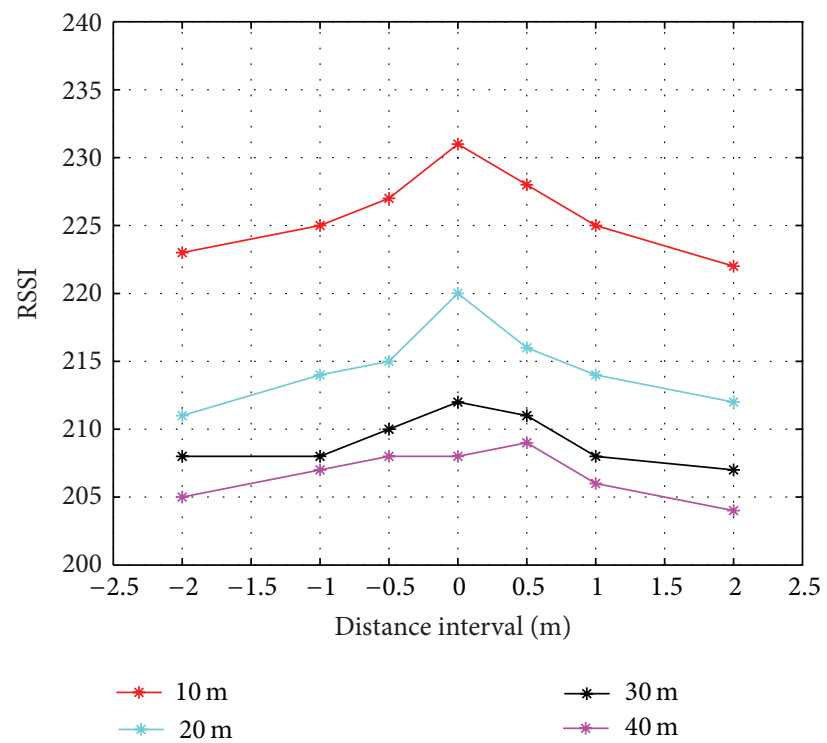

(c)

FIGURE 1: The experimental environment and result.

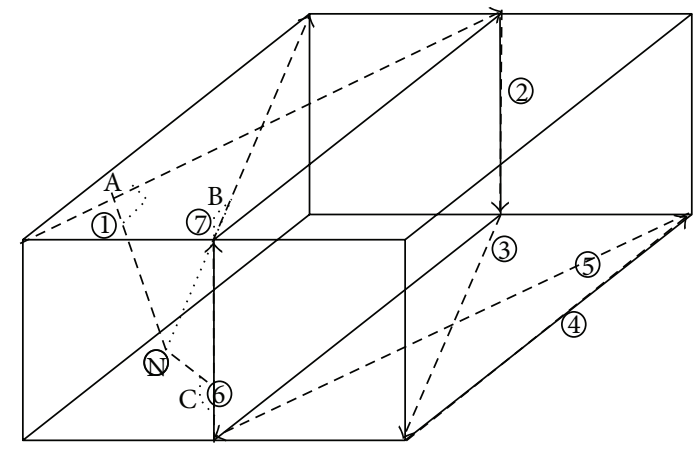

FIgUre 2: The model of HL.

As described in literature [34], the ratio of $\mathrm{AB}$ and $\mathrm{AD}$ should be $1: \sqrt{3}$. According to $(6)$,

$$
R_{\text {trusty }}: \mathrm{AE}: \mathrm{AB}: \mathrm{AD}=1: 1: 1: \sqrt{3} .
$$

The whole process of localization can be described as follows.

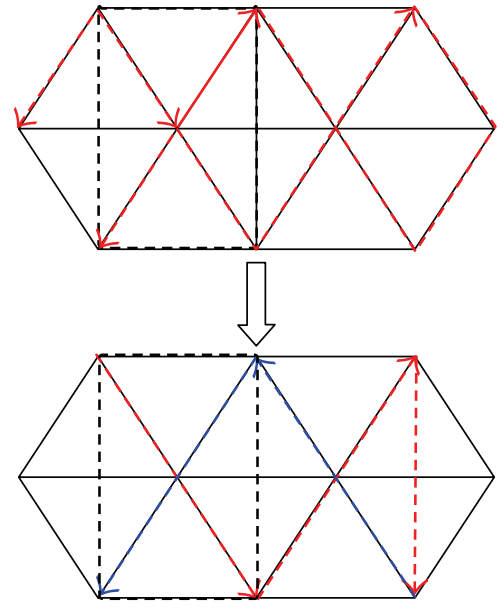

FIGURE 3: The transformation of the two-dimensional trajectory.

Step 1. The unknown nodes are randomly deployed in the three-dimensional space. 


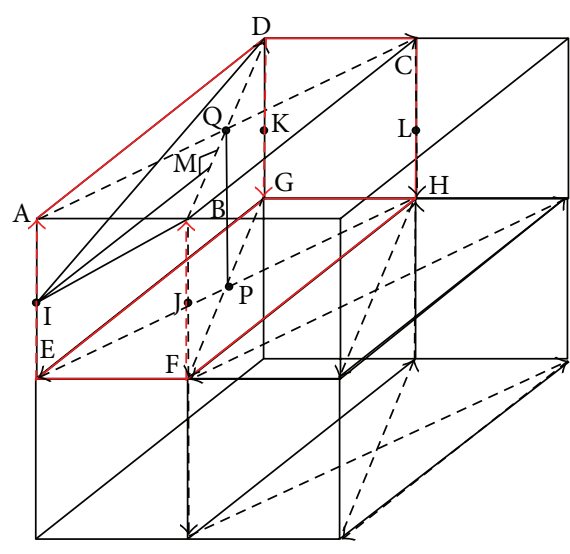

FIGURE 4: Example of the hexahedron.

TABLE 1: The trusty radius of different radii.

\begin{tabular}{lllllllll}
\hline Radius (m) & 30 & 40 & 50 & 60 & 70 & 80 & 90 & 100 \\
Trusty radius (m) & 20 & 25 & 30 & 35 & 40 & 45 & 50 & 60 \\
\hline
\end{tabular}

Step 2. The anchor moves along the trajectory according to the radius and broadcasts its location information and ID.

Step 3. The nodes receive and record information from the anchor (including signal strength).

Step 4. Each node finds out the nearest reference points on each of the three kinds of trajectory according to the RSSI.

Step 5. Implement the HL and calculate estimated position.

Step 6. The localization is finished.

The flow chart of IAPIT-3D is shown in Figure 5.

The Matlab pseudocode of localization period is displayed in Pseudocode 1.

\section{Simulation and Result}

In order to verify the theoretical feasibility of HL, the scientific tool MATLAB is adopted for the simulation. Take the conclusion of Section 3.1 for premise, we deploy 400 unknown nodes in the space of size $100 \mathrm{~m} \times 100 \mathrm{~m} \times 100 \mathrm{~m}$. The experiment is separately simulated by the moving step length of $1 \mathrm{~m}, 2 \mathrm{~m}, 3 \mathrm{~m}, 5 \mathrm{~m}, 6 \mathrm{~m}, 10 \mathrm{~m}$, and $15 \mathrm{~m}$. The result is described by the average absolute error and normalized average error (which is normalized to the ratio of the absolute average error to the radio range).

As Figures 6 and 7 show, the errors are increasing as the moving step length increases. In another word, the longer the length of step is, the more inaccurate the localization is. The reason is obvious that the shorter the step, is the more virtual beacon nodes are deployed. The location error is shown in Tables 2 and 3.

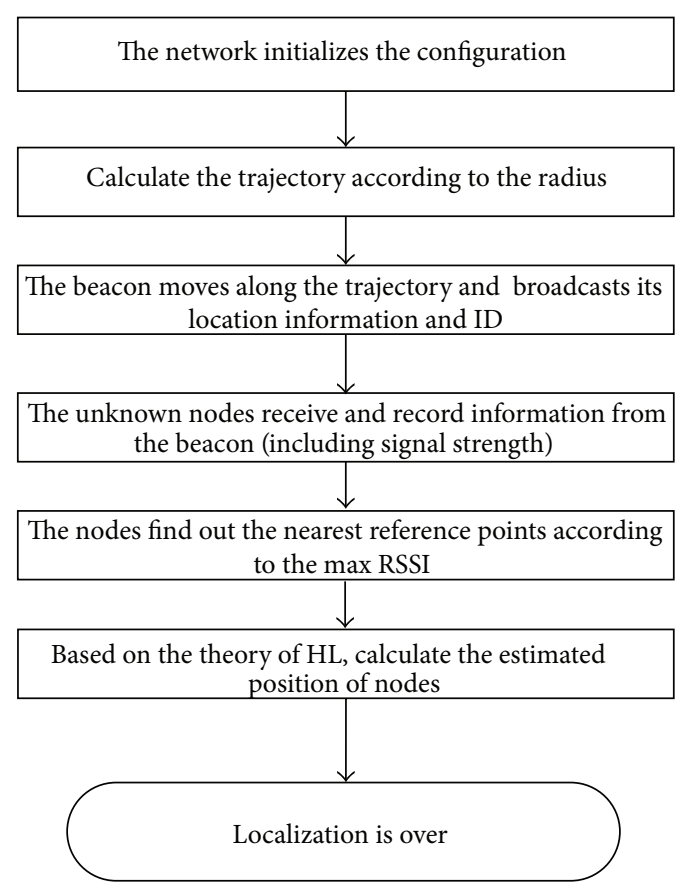

Figure 5: The flow chart of HL.

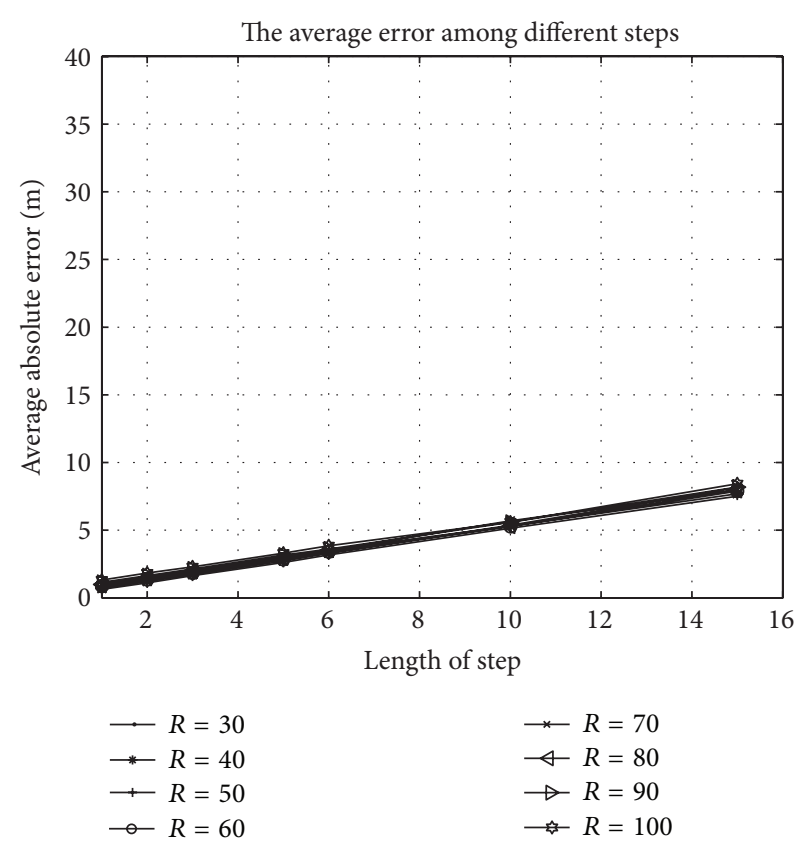

FIGURE 6: The average absolute error under different moving step lengths.

\section{The Relationship among the Parameters}

5.1. The Radius and the Absolute Error. In Figure 6, the seven curves that present absolute errors under different radius almost coincide. That means, as long as lengths of step are same, the absolute error is changeless although the radius is different. We can explain this phenomenon as follows: though radius is related to the deployment of virtual beacon, it does 


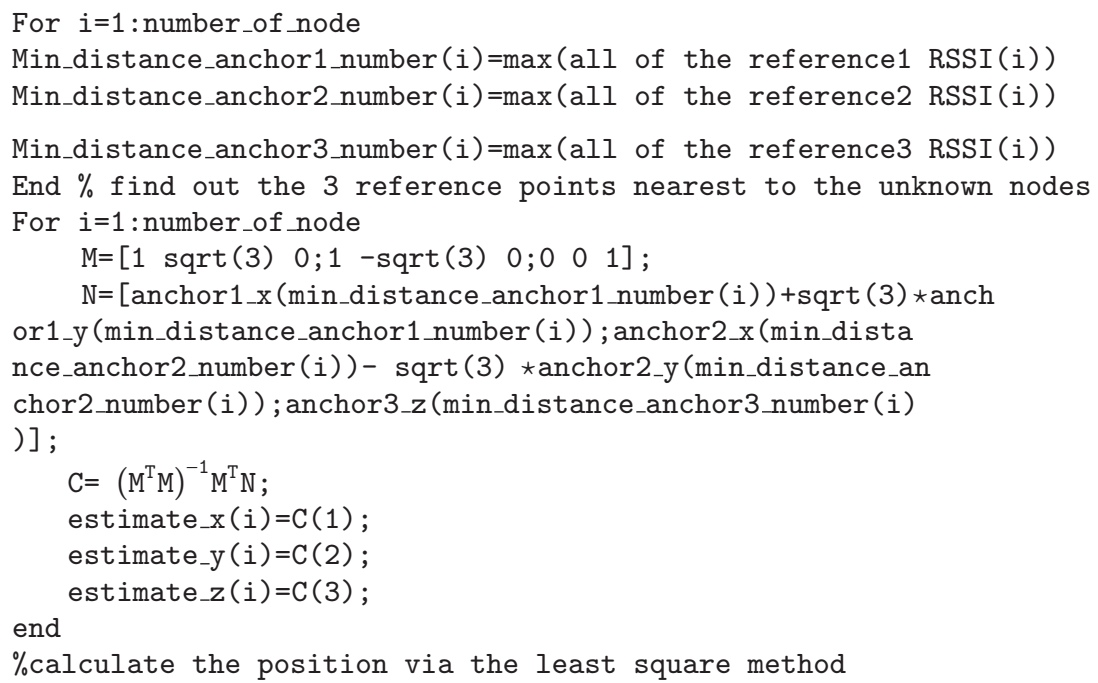

Pseudocode 1

TABLE 2: The absolute error $(\mathrm{m})$ under different radii and lengths of step.

\begin{tabular}{|c|c|c|c|c|c|c|c|}
\hline \multirow{2}{*}{$R(\mathrm{~m})$} & \multicolumn{7}{|c|}{ Step (m) } \\
\hline & 1 & 2 & 3 & 5 & 6 & 10 & 15 \\
\hline 30 & 0.63144 & 1.1046 & 1.6426 & 2.6104 & 3.1706 & 5.1427 & 7.5045 \\
\hline 40 & 0.68496 & 1.2053 & 1.7034 & 2.7165 & 3.2887 & 5.2988 & 7.6834 \\
\hline 50 & 0.7947 & 1.3097 & 1.7838 & 2.866 & 3.3216 & 5.3687 & 8.1604 \\
\hline 60 & 0.85757 & 1.3753 & 1.843 & 2.8639 & 3.4115 & 5.275 & 7.8843 \\
\hline 70 & 1.0429 & 1.5191 & 2.035 & 3.0941 & 3.5115 & 5.6858 & 7.9872 \\
\hline 80 & 0.99774 & 1.5054 & 1.9853 & 2.9654 & 3.5361 & 5.3063 & 7.9795 \\
\hline 90 & 1.1535 & 1.6372 & 2.1323 & 3.1556 & 3.5957 & 5.5929 & 8.1876 \\
\hline 100 & 1.3238 & 1.831 & 2.2867 & 3.3089 & 3.8477 & 5.5941 & 8.424 \\
\hline
\end{tabular}

TABLE 3: The normalized error under different radii and length of step.

\begin{tabular}{lccccccc}
\hline$R(\mathrm{~m})$ & \multicolumn{5}{c}{ Step $(\mathrm{m})$} & 5 & 10 \\
\hline 30 & 1 & 2 & 3 & 5 & 8.7014 & 10.569 & 17.142 \\
40 & 2.1048 & 3.682 & 5.4753 & 6.7911 & 8.2217 & 13.247 \\
50 & 1.7124 & 3.0133 & 4.2585 & 5.732 & 6.6431 & 10.737 & 19.209 \\
60 & 1.5894 & 2.6193 & 3.5677 & 4.7731 & 5.6858 & 8.7917 & 16.321 \\
70 & 1.4293 & 2.2922 & 3.0717 & 4.4201 & 5.0164 & 8.1226 \\
80 & 1.4899 & 2.1701 & 2.9071 & 3.7068 & 4.4201 & 6.6329 \\
90 & 1.2472 & 1.8818 & 2.4816 & 3.5062 & 3.9952 & 6.2144 \\
100 & 1.2816 & 1.8191 & 2.3692 & 3.3089 & 3.8477 & 9.9744 \\
\hline
\end{tabular}

not influence the trusty coverage of mobile beacon in the total space. In another word, the change of radius cannot effect whether unknown nodes are covered in the trusty communication extension as long as the step of movement is definite. From Table 2, we can discover that the absolute error is increasing when $R$ increases. However, the influence of radius to the error is negligible relative to that of step length.
We depict the relationship among radius, length of step, and the absolute error in a 3D picture. In Figure 8, the $x$-label and the $y$-label present radius and length of step. And the $z$ label presents the absolute error of the localization. From the piecemeal change of the color in Figure 8, we find that the influence of step length to the absolute error is greater than that of radius. 


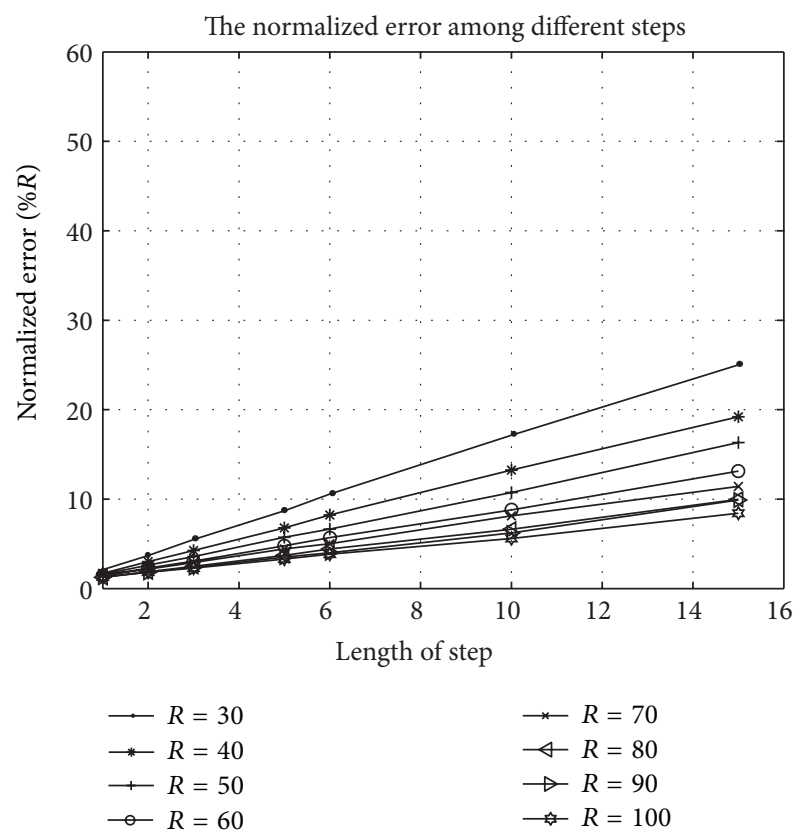

FIGURE 7: The average normalized error under different moving step lengths.

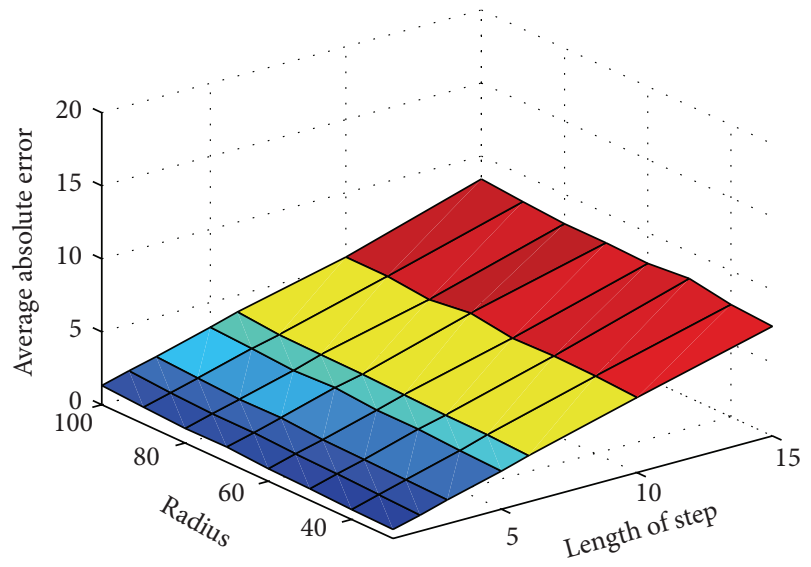

FIgURE 8: The 3D picture of the absolute error in localization.

5.2. The Radius and the Normalized Error. The relationship between radius and the normalized error is shown in Figure 6 and Table 3. Different from Figure 6, we find that the normalized error is decreasing when the radius increases. The $3 \mathrm{D}$ picture of the normalized error is shown in Figure 9.

Normalized error is the ratio of the absolute error to the radius. As the absolute error increases, the radius increases. This phenomenon leads to a problem of balancing between radius and accuracy. In localization of WSN, there are two principle elements to be paid attention to: (1) the cost; (2) the accuracy and the precision [40]. In our scheme, the cost is reflected by the radius to a certain extent. Communication cost is influenced by two points: first, the size of model, which affects the number of the packages sent. Second, it is the transmitting power. And these two points are all relative

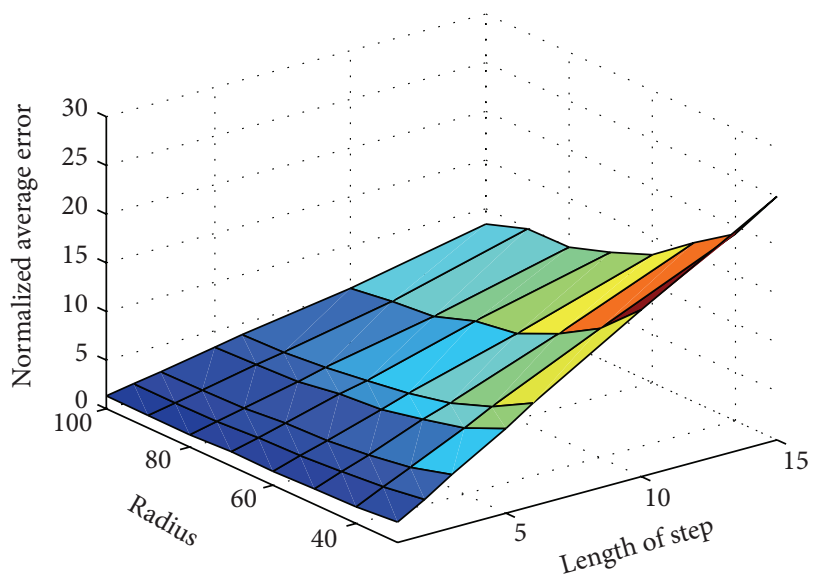

FIgURE 9: The 3D picture of the normalized error in the localization.

to the radius. Next, we will analyze the relationship among these elements. Take moving step of $1 \mathrm{~m}$ for an example, the relationship between radius and number of packages sent is shown in Table 4.

According to the formula of the signal space loss,

$$
[\mathrm{Lfs}](\mathrm{dB})=32.44+20 \lg d(\mathrm{~km})+20 \lg f(\mathrm{MHz}) .
$$

The following equations should be established:

$$
\begin{aligned}
\operatorname{Lfs}(d) & =32.44+20 \lg (0.03)+20 \lg f+20 \lg \left(\frac{d}{0.03}\right) \\
& =\operatorname{Lfs}(0.03)+20 \lg \left(\frac{d}{0.03}\right) .
\end{aligned}
$$

In the light of the operating frequency $=2.4 \mathrm{GHz}$ and the receiving sensitivity $=-105 \mathrm{dBm}$ of the CC2420 receiver, and the transmitting power is $W_{1}=-35(\mathrm{dBm})$ when the radius is $30 \mathrm{~m}$, the transmitting powers under different radius are shown in Table 5.

The formula of transforming $\mathrm{dBm}$ to mw obeys this equation:

$$
x(\mathrm{dBm})=10 \lg [p(\mathrm{mw})] .
$$

Combined with Table 4, we show the ratio of consumed energy under the same step length in Table 6 .

As described in Table 6, it is obvious that the consumed energy is lowest when the radius is $30 \mathrm{~m}$. So, the performance is increasingly excellent as the radius is reducing. At this moment, the traditional normalized error is meaningless. However, there is a drawback that the trajectory is less controllable in the short-radius localization of our scheme.

5.3. The Step Length and the Absolute Error. Taking $R=100 \mathrm{~m}$ for an example, the relationship between moving step and number of packages send is shown in Table 7 .

According to the ratio of the message package numbers, we can get the approximate ratio of the consumed energy with different step length. Because the consumed energy of every package is equal, the product of absolute error and number 
TABLE 4: Relationship between radius and number of the packages sent.

\begin{tabular}{lc}
\hline Radius $(\mathrm{m})$ & Number of the packages \\
\hline 30 & 13224 \\
40 & 9075 \\
50 & 4888 \\
60 & 3414 \\
70 & 3882 \\
80 & 4350 \\
90 & 2348 \\
100 & 1282 \\
\hline
\end{tabular}

TABLE 5: The transmitting powers under different radii (step length $=1 \mathrm{~m}$ ).

\begin{tabular}{lc}
\hline Radius $(\mathrm{m})$ & Transmitting power $(\mathrm{dBm})$ \\
\hline 30 & -35.4134 \\
40 & -32.9146 \\
50 & -30.9764 \\
60 & -29.3928 \\
70 & -28.0538 \\
80 & -26.8940 \\
90 & -25.8709 \\
100 & -24.9558 \\
\hline
\end{tabular}

TABLE 6: The ratio of consumed energy under the same step length.

\begin{tabular}{lc}
\hline Radius $(\mathrm{m})$ & Consumed energy \\
\hline 30 & 3.8021 \\
40 & 4.6386 \\
50 & 3.9038 \\
60 & 3.9263 \\
70 & 6.0768 \\
80 & 8.8938 \\
90 & 6.0759 \\
100 & 4.0955 \\
\hline
\end{tabular}

TABLE 7: Relationship between moving step and number of the packages sent $(R=100 \mathrm{~m})$.

\begin{tabular}{lc}
\hline Step length $(\mathrm{m})$ & Number of the packages \\
\hline 1 & 1282 \\
2 & 641 \\
3 & 427 \\
5 & 257 \\
6 & 214 \\
10 & 128 \\
15 & 86 \\
\hline
\end{tabular}

of the package can be a norm to reflect the relative error of the localization under the same radius but with different step lengths. When $R=100 \mathrm{~m}$, the relative error is shown in Table 8.

On the basis of Table 8, we find that the relative error is the lowest when the step length is $10 \mathrm{~m}$. So, $10 \mathrm{~m}$ is the best length
TABLE 8: The relative error under different step lengths $(R=100 \mathrm{~m})$.

\begin{tabular}{lc}
\hline Step length $(\mathrm{m})$ & Relative error \\
\hline 1 & 1697.1 \\
2 & 1173.7 \\
3 & 976.42 \\
5 & 850.39 \\
6 & 823.41 \\
10 & 716.04 \\
15 & 724.46 \\
\hline
\end{tabular}

TABLE 9: The theoretically absolute error under different length of step.

\begin{tabular}{lc}
\hline Step length $(\mathrm{m})$ & Absolute error $(\mathrm{m})$ \\
\hline 1 & 1.1180 \\
2 & 2.2361 \\
3 & 3.3541 \\
5 & 5.5902 \\
6 & 6.7082 \\
10 & 11.1803 \\
15 & 16.7705 \\
\hline
\end{tabular}

of steps to get the proper accuracy under relative smaller overhead of communication when $R=100 \mathrm{~m}$.

\section{The Error Analysis}

The error in the process of localization is an important criterion of the locating performance. The goal of error analysis is finding out the "source of error" in order to improve and optimize the schemes in the future work. It is usually that errors are caused by various elements. In this section, we will analyze the main element that leads to the ultimate error of the localization. In our scheme, the largest error is aroused in the process of least square method. The reason is that the referenced point may not be the exact foot point of the trajectory. The error starts at the selection of reference point. Assume that, the length of moving step is $a$, the distance between the foot of the trajectory's perpendicular and the reference point $\Delta$ is in the interval $[0, a / 2]$. If the coordinates of the reference points are

$$
\left(x_{a}, y_{a}, z_{a}\right)\left(x_{b}, y_{b}, z_{b}\right)\left(x_{c}, y_{c}, z_{c}\right)
$$

and the coordinates of the foot of the trajectory's perpendicular are

$$
\left(x_{a}^{\prime}, y_{a}^{\prime}, z_{a}^{\prime}\right)\left(x_{b}^{\prime}, y_{b}^{\prime}, z_{b}^{\prime}\right)\left(x_{c}^{\prime}, y_{c}^{\prime}, z_{c}^{\prime}\right)
$$

As described in Section 3, the direction vectors are

$$
\begin{gathered}
\left(i_{1}, j_{1}, k_{1}\right)=(1, \sqrt{3}, 0), \\
\left(i_{2}, j_{2}, k_{2}\right)=(-1, \sqrt{3}, 0), \\
\left(i_{3}, j_{3}, k_{3}\right)=(0,0,1) .
\end{gathered}
$$


TABLE 10: The theoretically normalized error under different radii and lengths of step.

\begin{tabular}{|c|c|c|c|c|c|c|c|}
\hline \multirow{2}{*}{$R(\mathrm{~m})$} & \multicolumn{7}{|c|}{ Step (m) } \\
\hline & 1 & 2 & 3 & 5 & 6 & 10 & 15 \\
\hline 30 & 3.73 & 7.45 & 11.18 & 18.63 & 22.36 & 37.27 & 55.90 \\
\hline 40 & 2.80 & 5.59 & 8.39 & 13.98 & 16.77 & 27.95 & 41.93 \\
\hline 50 & 2.24 & 4.47 & 6.71 & 11.18 & 13.42 & 22.36 & 33.54 \\
\hline 60 & 1.86 & 3.73 & 5.59 & 9.32 & 11.18 & 18.63 & 27.95 \\
\hline 70 & 1.60 & 3.19 & 4.79 & 7.99 & 9.58 & 15.97 & 23.96 \\
\hline 80 & 1.40 & 2.80 & 4.19 & 6.99 & 8.39 & 13.98 & 20.96 \\
\hline 90 & 1.24 & 2.48 & 3.73 & 6.21 & 7.45 & 12.42 & 18.63 \\
\hline 100 & 1.12 & 2.24 & 3.35 & 5.59 & 6.71 & 11.18 & 16.77 \\
\hline
\end{tabular}

So, (3) is converted into

$$
\begin{aligned}
& \left(\begin{array}{l}
x \\
y \\
z
\end{array}\right)=\left(\begin{array}{ccc}
\frac{1}{2} & -\frac{1}{2} & 0 \\
\frac{\sqrt{3}}{6} & \frac{\sqrt{3}}{6} & 0 \\
0 & 0 & 1
\end{array}\right)\left(\begin{array}{c}
x_{a}+\sqrt{3} y_{a} \\
-x_{b}+\sqrt{3} y_{b} \\
z_{c}
\end{array}\right) \\
& \Longrightarrow\left(\begin{array}{l}
x \\
y \\
z
\end{array}\right)=\left(\begin{array}{c}
\frac{\left(x_{a}+x_{b} \sqrt{3} y_{a}-\sqrt{3} y_{b}\right)}{2} \\
\frac{\sqrt{3}\left(x_{a}-x_{b}+\sqrt{3} y_{a}+\sqrt{3} y_{b}\right)}{6}
\end{array}\right) .
\end{aligned}
$$

As to the specific size of the model, the relationship between the foot of the trajectory's perpendicular and the reference point is revealed as follows:

$$
\left(\begin{array}{ccc}
x_{a} & y_{a} & z_{a} \\
x_{b} & y_{b} & z_{b} \\
x_{c} & y_{c} & z_{c}
\end{array}\right)=\left(\begin{array}{ccc}
x_{a}^{\prime}+\frac{\Delta}{2} & y_{a}^{\prime}+\frac{\sqrt{3} \Delta}{2} & z_{a} \\
x_{b}^{\prime}+\frac{\Delta}{2} & y_{b}^{\prime}-\frac{\sqrt{3} \Delta}{2} & z_{b} \\
x_{c} & y_{c} & z_{c}^{\prime}+\Delta
\end{array}\right) .
$$

Equation (15) should be transformed into

$$
\left(\begin{array}{l}
x \\
y \\
z
\end{array}\right)=\left(\begin{array}{c}
\frac{\left(x_{a}^{\prime}+x_{b}^{\prime}+\sqrt{3} y_{a}^{\prime}-\sqrt{3} y_{b}^{\prime}\right)}{2}+2 \Delta \\
\frac{\sqrt{3}\left(x_{a}^{\prime}-x_{b}^{\prime}+\sqrt{3} y_{a}^{\prime}+\sqrt{3} y_{b}^{\prime}\right)}{6} \\
z_{c}^{\prime}+\Delta
\end{array}\right) .
$$

According to the above, the ultimate error is

$$
\sqrt{(2 \Delta)^{2}+(\Delta)^{2}}=\sqrt{5} \Delta
$$

owing to the

$$
\Delta \in\left[0, \frac{a}{2}\right]
$$

the theoretical maximum ultimate error

$$
\begin{gathered}
\max \left(\text { error }_{\text {absolute }}\right)=\frac{\sqrt{5}}{2} a, \\
\max \left(\text { error }_{\text {normalized }}\right)=\frac{\sqrt{5 a}}{2 R} .
\end{gathered}
$$

In Table 2, we discovered that a few of errors are greater than the theoretical value as shown in Tables 9 and 10. This is caused by the imperfection of the model. The balance of size of the space and the model is also a key point of the localization here.

\section{Conclusion and Future Work}

$\mathrm{HL}$ is a range-free localization scheme and can be applied under the case that the hardware is relatively limited. We use the special property of trajectory's perpendicular to calculate the coordinate of the unknown nodes. The trajectory is optimized via the geometry constraint, and the locating process is simulated by the tool of MATLAB. The performance of $\mathrm{HL}$ is relative perfect in aspect of accuracy. We compare the accuracy under different radius and obtain the coarse bound of the suitable radii. However, the mobile beacon's trajectory may be uncontrollable, and the trajectory may not be the ultimate one. In the next period of work, we shall devote ourselves to studying in the research of model optimization.

\section{Acknowledgments}

The authors would like to thank Nataliya Shapovalova (Autonomous University of Barcelona, Spain) for providing us with fruitful comments which significantly increased the quality of the paper and Zhanwu Xiong (Autonomous University of Barcelona, Spain) for developing suitable software tools. This paper is sponsored by the National Nature Science Foundation of China (no. 61363015, no. 61262020), Aeronautical Science Foundation of China (2012ZC56006), and Key Project of Research Program of Jiangxi Province (CB201120382).

\section{References}

[1] S. Limin, L. Jian, and Y. Chen, Wireless Sensor Networks: The Complete Book, Tsinghua University Press, Beijing, China, 2005.

[2] Y. Liu, Z. Yang, X. Wang, and L. Jian, "Location, localization, and localizability," Journal of Computer Science and Technology, vol. 25, no. 2, pp. 274-297, 2010.

[3] H. Chen, P. Huang, M. Martins, H. C. So, and K. Sezaki, "Novel centroid localization algorithm for three-dimensional wireless 
sensor networks," in Proceedings of the IEEE International Conference on Wireless Communications, Networking and Mobile Computing (WiCOM '08), pp. 1-4, Dalian, China, October 2008.

[4] N. Bulusu, J. Heidemann, and D. Estrin, "Density adaptive algorithms for beacon placement in wireless sensor networks," in Proceedings of the IEEE Conference on Distributed Computing Systems (ICDCS '01), Phoenix, Ariz, USA, April 2001.

[5] J. Wang, P. Urriza, Y. Han, and D. Cabric, "Weighted centroid localization algorithm: theoretical analysis and distributed implementation," IEEE Transactions on Wireless Communications, vol. 10, no. 10, pp. 3403-3413, 2011.

[6] J. Z. Wang and H. Jin, "Improvement on APIT localization algorithms for wireless sensor networks," in Proceedings of the International Conference on Networks Security, Wireless Communications and Trusted Computing (NSWCTC '09), pp. 719-723, Wuhan, China, April 2009.

[7] T. He, C. Huang, B. M. Blum, J. A. Stankovic, and T. Abdelzaher, "Range-free localization schemes for large scale sensor networks," in Proceedings of the 9th Annual International Conference on Mobile Computing and Networking (MobiCom '03), pp. 81-95, San Diego, Calif, USA, 2003.

[8] J. Zhao, Q. Pei, and Z. Xu, "APIT localization algorithms for wireless sensor networks," Computer Engineering, vol. 33, no. 5, pp. 109-111, 2007.

[9] H. Chen, P. Huang, M. Martins, H. C. So, and K. Sezaki, "Novel centroid localization algorithm for three-dimensional wireless sensor networks," in Proceedings of the IEEE International Conference on Wireless Communications, Networking and Mobile Computing (WiCOM '08), vol. 4, Dalian, China, October 2008.

[10] J. Yang and F. Liu, "A modified localization algorithm of apit based on perpendicular bisector feature for wireless sensor network," Chinese Journal of Sensors and Actuators, vol. 21, no. 8, pp. 1453-1457, 2008.

[11] Y. Liu, J. Pu, Y. He, and Z. Xiong, "Three-dimensional selflocalization scheme for wireless sensor networks," Journal of Beijing University of Aeronautics and Astronautics, vol. 34, no. 6, pp. 647-651, 2008.

[12] J. Shu, L. Liu, Y. Chen, and H. Hu, "A novel three-dimensional localization algorithm in wireless sensor networks," in Proceedings of the 4th International Conference on Wireless Communications Networking and Mobile Computing (WiCOM '08), pp. 1-3, Dalian, China, 2008.

[13] N. Bulusu, J. Heidemann, and D. Estrin, "GPS-less low-cost outdoor localization for very small devices," IEEE Personal Communications, vol. 7, no. 5, pp. 28-34, 2000.

[14] Z. Dengyi and L. Feng, "Improvement of DV-Hop localization algorithms in wireless sensor networks," in Proceedings of the IEEE International Symposium on Instrumentation \& Measurement, Sensor Network and Automation (IMSNA '12), pp. 567569, 2012.

[15] M. T. Isik and O. B. Akan, "A three dimensional localization algorithm for underwater acoustic sensor networks," IEEE Transactions on Wireless Communications, vol. 8, no. 9, pp. 4457-4463, 2009.

[16] L. Girod and D. Estrin, "Robust range estimation using acoustic and multimodal sensing," in Proceedings of the IEEE/RSJ International Conference on Intelligent Robotsand Systems (IROS'01), vol. 3, pp. 1312-1320, Maui, Hawaii, USA, 2001.

[17] X. Y. Li, L. L. Ci, G. Y. Ge, and D. W. Li, "Acoustic source localization via advanced TDoA for self-organization wireless sensor networks," Applied Mechanics and Materials, vol. 50, pp. 42-48, 2011.
[18] N. B. Priyantha, A. Chakraborty, and H. Balakrishnan, "The cricket location-support system," in Proceedings of the 6th ACM International Conference on Mobile Computing and Networking (ACM MOBICOM '00), pp. 32-43, Boston, Mass, USA, August 2000.

[19] A. Savvides, C.-C. Han, and B. M. Strivastava, "Dynamic finge-grained localization in ad-hoc networks of sensor," in Proceedings of the 7th Annual International Conference on Mobile Computing and Networking (MobiCom '01), pp. 166-179, Rome, Italy, July 2001.

[20] A. Savvides, H. Park, and M. B. Srivastava, "The bits and flops of the n-hop multilateration primitive for node localization problems," in Proceedings of the 1st ACM International Workshop on Wireless Sensor Networks and Applications, pp. 112-121, Atlanta, Ga, USA, September 2002.

[21] H. S. AbdelSalam and S. Olariu, "A 3d-localization and terrain modeling technique for wireless sensor networks," in Proceedings of the 2nd ACM International Workshop on Foundations of Wireless Ad Hoc and Sensor Networking and Computing (FOWANC’09), pp. 37-46, New York, NY, USA, May 2009.

[22] K. Benkič, M. Malajner, P. Planinšič, and Ž. Čučej, "Using RSSI value for distance estimation in wireless sensor networks based on Zig-Bee," in Proceedings of the 15th International Conference on Systems, Signals and Image Processing (IWSSIP '08), pp. 303306, Bratislava, Slovakia, June 2008.

[23] A. Nafarieh and J. Ilow, "A testbed for localizing wireless LAN devices using received signal strength," in Proceedings of the 6th Annual Communication Networks and Services Research Conference (CNSR '08), pp. 481-487, Halifax, Canada, May 2008.

[24] X. Shen, Z. Wang, P. Jiang, R. Lin, and Y. Sun, "Connectivity and RSSI based localization scheme for wireless sensor networks," in Advances in Intelligent Computing, vol. 3645 of Lecture Notes in Computer Science, pp. 578-587, Springer, Berlin, Germany, 2005.

[25] C.-W. Fan, Y.-H. Wu, and W.-M. Chen, "RSSI-based localization for wireless sensor networks with a mobile beacon," in Proceedings of the IEEE Sensors, pp. 1-4, Taipei, Taiwan, October 2012.

[26] M. L. Sichitiu and V. Ramadurai, "Localization of wireless sensor networks with a mobile beacon," in Proceedings of the IEEE International Conference on Mobile Ad-Hoc and Sensor Systems (MAHSS '04), pp. 174-183, October 2004.

[27] K.-F. Ssu, C.-H. Ou, and H. C. Jiau, "Localization with mobile anchor points in wireless sensor networks," IEEE Transactions on Vehicular Technology, vol. 54, no. 3, pp. 1187-1197, 2005.

[28] C.-H. Ou, K.-F. Ssu, and H. C. Jiau, "Range-free localization with aerial anchors in wireless sensor networks," International Journal of Distributed Sensor Networks, vol. 2, no. 1, pp. 1-21, 2006.

[29] D. Koutsonikolas, S. M. Das, and Y. C. Hu, "Path planning of mobile landmarks for localization in wireless sensor networks," Computer Communications, vol. 30, no. 13, pp. 2577-2592, 2007.

[30] G. Yu, F. Yu, and L. Feng, "A three dimensional localization algorithm using a mobile anchor node under wireless channel," in Proceedings of the IEEE International Joint Conference on Neural Networks (IJCNN '08), pp. 477-483, Hong Kong, June 2008.

[31] B. Zhang, F. Yu, and Z. Zhang, "An improved localization algorithm for wireless sensor network using a mobile anchor node," in Proceedings of the IEEE Asia-Pacific Conference on Information Processing (APCIP '09), pp. 485-488, Shenzhen, China, July 2009. 
[32] S. Lee, E. Kim, C. Kim, and K. Kim, "Localization with a mobile beacon based on geometric constraints in wireless sensor networks," IEEE Transactions on Wireless Communications, vol. 8, no. 12, pp. 5801-5805, 2009.

[33] H. Li, Y. Bu, H. Xue, X. Li, and H. Ma, "Path planning for mobile anchor node in localization for wireless sensor networks," Computer Research and Development, vol. 46, no. 1, pp. 129-136, 2009.

[34] Z. Guo, Y. Guo, F. Hong et al., "Perpendicular intersection: locating wireless sensors with mobile beacon," IEEE Transactions on Vehicular Technology, vol. 59, no. 7, pp. 3501-3509, 2010.

[35] C. H. Ou, "A localization scheme for wireless sensor networks using mobile anchors with directional antennas," IEEE Sensors Journal, vol. 11, no. 7, pp. 1607-1616, 2011.

[36] X. Li, N. Mitton, I. Simplot-Ryl, and D. Simplot-Ryl, "Dynamic beacon mobility scheduling for sensor localization," IEEE Transactions on Parallel and Distributed Systems, vol. 23, no. 8, pp. 1439-1452, 2012.

[37] C.-T. Chang, C.-Y. Chang, and C.-Y. Lin, "Anchor-guiding mechanism for beacon-assisted localization in wireless sensor networks," IEEE Sensors Journal, vol. 12, no. 5, pp. 1098-1111, 2012.

[38] Y. S. Chen, Y. J. Ting, C. H. Ke, N. Chilamkurti, and J. H. Park, "Efficient localization scheme with ring overlapping by utilizing mobile anchors in wireless sensor networks," ACM Transactions on Embedded Computing Systems, vol. 12, no. 2, article 20, 2013.

[39] T. S. Rappaport, Wireless Communications Principle and Practical Application: The Complete Book, Publishing House of Electrinics Industry, Beijing, China, 2006.

[40] M. Bal, L. Min, S. Weiming, and G. Hamada, "Localization in cooperative wireless sensor networks: a review," in Proceedings of the 13th International Conference on Computer Supported Cooperative Work in Design (CSCWD '09), pp. 438-443, Santiago, Chile, April 2009. 

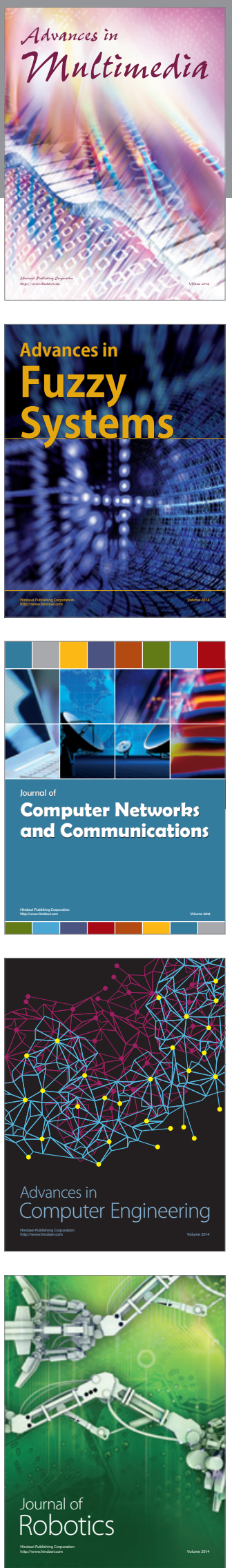

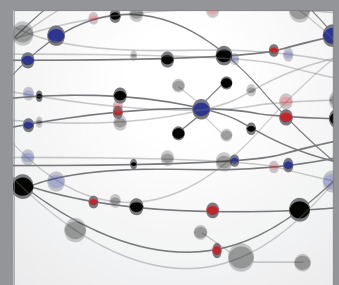

The Scientific World Journal
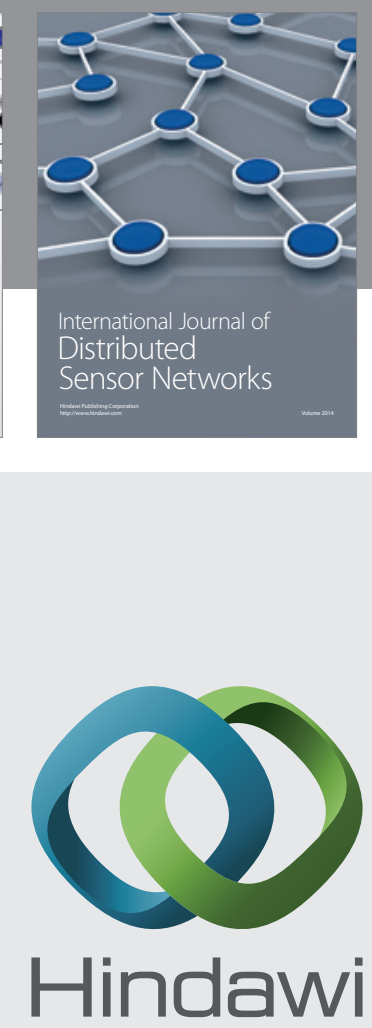

Submit your manuscripts at

http://www.hindawi.com
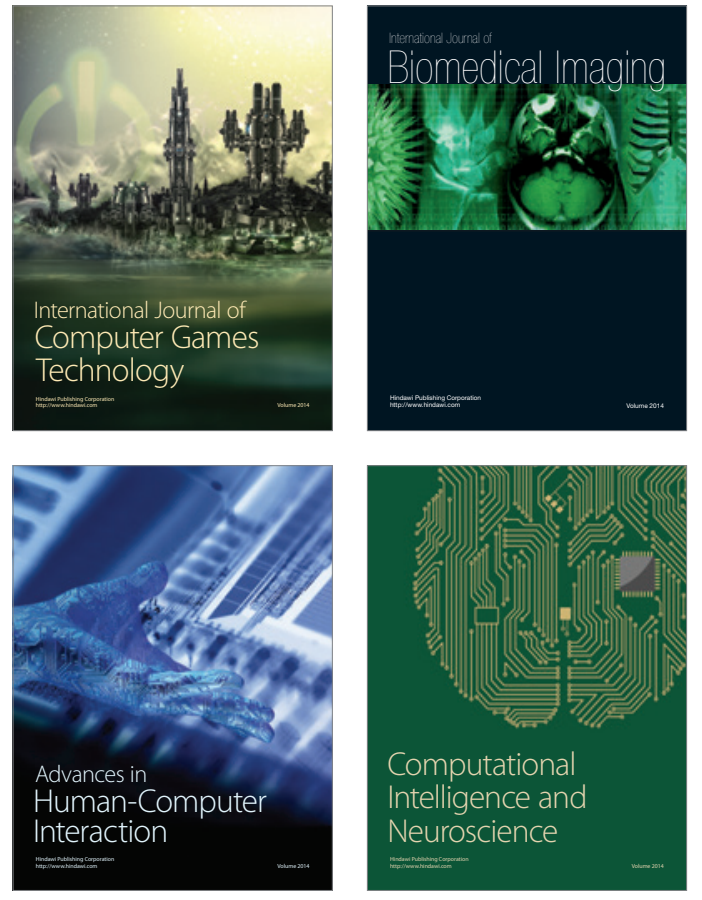
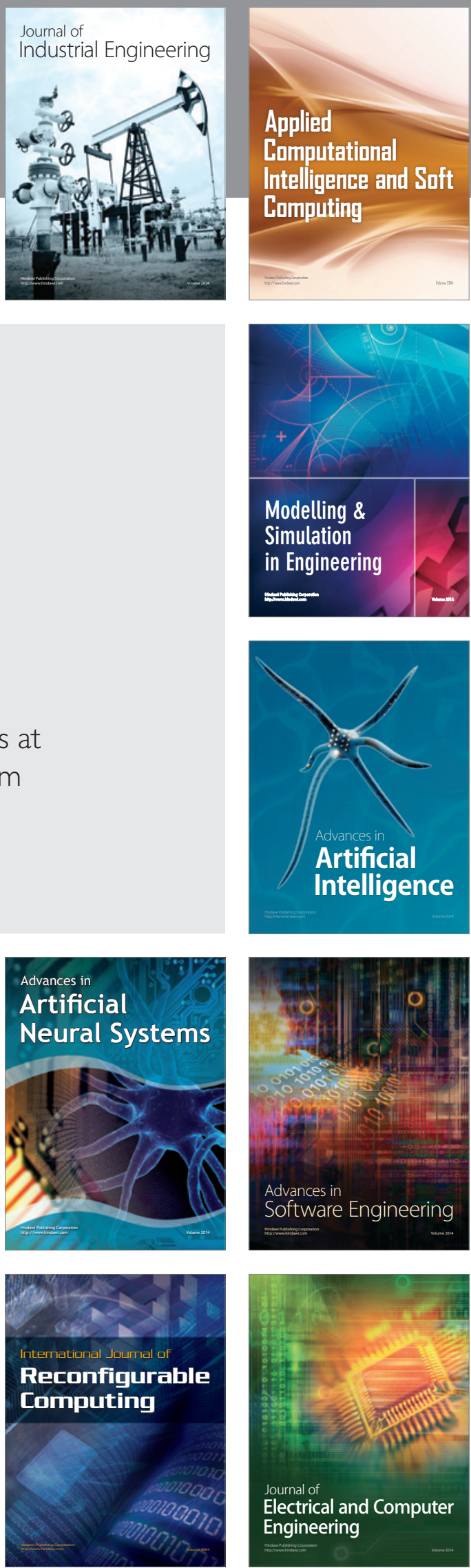